ARTICLE

DOI: $10.1038 / s 41467-018-04175-y$

\title{
CRISPR-LbCpf1 prevents choroidal neovascularization in a mouse model of age-related macular degeneration
}

Taeyoung Koo ${ }^{1,2}$, Sung Wook Park (10 3,4,5, Dong Hyun Jo (10 ${ }^{3}$, Daesik Kim ${ }^{6}$, Jin Hyoung Kim ${ }^{3}$, Hee-Yeon Cho ${ }^{1}$, Jeungeun $\mathrm{Kim}^{6}$, Jeong Hun Kim ${ }^{3,4,5}$ \& Jin-Soo Kim ${ }^{1,2,6}$

LbCpf1, derived from Lachnospiraceae bacterium ND2006, is a CRISPR RNA-guided endonuclease and holds promise for therapeutic applications. Here we show that LbCpf1 can be used for therapeutic gene editing in a mouse model of age-related macular degeneration (AMD). The intravitreal delivery of $\mathrm{LbCpf1}$, targeted to two angiogenesis-associated genes encoding vascular endothelial growth factor A (Vegfa) and hypoxia inducing factor 1a (Hif1a), using adeno-associated virus, led to efficient gene disruption with no apparent off-target effects in the retina and retinal pigment epithelium (RPE) cells. Importantly, LbCpf1 targeted to Vegfa or Hifla in RPE cells reduced the area of laser-induced choroidal neovascularization as efficiently as aflibercept, an anti-VEGF drug currently used in the clinic, without inducing cone dysfunction. Unlike aflibercept, LbCpf1 targeted to Vegfa or Hifla achieved a long-term therapeutic effect on CNV, potentially avoiding repetitive injections. Taken together, these results indicate that LbCpf1-mediated in vivo genome editing to ablate pathologic angiogenesis provides an effective strategy for the treatment of AMD and other neovascularization-associated diseases.

\footnotetext{
${ }^{1}$ Center for Genome Engineering, Institute for Basic Science, Seoul 151-747, Republic of Korea. ${ }^{2}$ Department of Basic Science, University of Science and Technology, Daejeon 34113, Republic of Korea. ${ }^{3}$ FARB Laboratory, Biomedical Research Institute, Seoul National University Hospital, Seoul 03082, Republic of Korea. ${ }^{4}$ Department of Biomedical Sciences, Seoul National University College of Medicine, Seoul, Republic of Korea. ${ }^{5}$ Department of Ophthalmology, Seoul National University College of Medicine, Seoul 03080, Republic of Korea. ${ }^{6}$ Department of Chemistry, Seoul National University, Seoul 151-747, South Korea. These authors contributed equally: Taeyoung Koo, Sung Wook Park. Correspondence and requests for materials should be addressed to J.H.K. (email: steph25@snu.ac.kr) or to J.-S.K. (email: jskim01@snu.ac.kr)
} 
W et age-related macular degeneration (AMD), a neovascular disease that leads to vision loss in the center of the visual field, occurs most commonly in individuals over the age of $50^{1}$. Several angiogenic growth factors in the retina have been implicated, including vascular endothelial growth factor $(\mathrm{VEGF})^{2}$. The advent of anti-VEGF agents (e.g., aflibercept, bevacizumab, and ranibizumab) has led to significantly reduced retinal and choroidal neovascularizations (CNV) in the clinic ${ }^{3,4}$. However, currently used anti-VEGF therapies require frequent repetitive injections over time to sustain the therapeutic effect on ocular neovascularization ${ }^{5-7}$. Therefore, it is ideal to suppress pathologic angiogenesis with a single treatment over the long-term.

The type II CRISPR (clustered regularly interspaced short palindromic repeats) system, an adaptive immune system used by bacteria and archaea to defend against viral infection ${ }^{8}$, has been repurposed for site-specific genome modification ${ }^{9-12}$ and become a promising approach for the treatment of diverse genetic or nongenetic disorders ${ }^{13-16}$. The Cas9 protein (SpCas9), derived from Streptococcus pyogenes, in combination with a small-guide RNA (sgRNA), a synthetic fusion of CRISPR RNA (crRNA) and transactivating crRNA (tracrRNA) ${ }^{17}$, recognizes and cleaves a target locus, generating a blunt-ended double-stranded break (DSB) three nucleotides upstream of a protospacer adjacent motif (PAM). However, the relatively large size of the SpCas9-coding sequence (4.10 kbp encoding 1,368 amino acids) makes it difficult or impossible to package the system with a sgRNA expression cassette into an adeno-associated virus (AAV). Furthermore, concerns remain about SpCas9 off-target nuclease activity. These issues have accelerated efforts to develop alternative genome editing tools.

A recently reported RNA-guided endonuclease, CRISPR from Prevoltella and Francisella 1 (Cpf1), is derived from a type V (class II) CRISPR system and differs from Cas9 in several key respects ${ }^{18}$. Cpf1 is guided by a single crRNA, without the need for a tracrRNA ${ }^{18}$. In contrast to Cas9, Cpf1 exhibits ribonuclease activity, enabling it to process its precursor crRNA into mature crRNAs ${ }^{19}$. Cpf1 recognizes a T-rich PAM (5'-TTTV-3') at the $5^{\prime}$ - end of a protospacer; DNA cleavage generates a staggered DSB distal to the PAM site ${ }^{20}$. It has been reported that LbCpf1 from Lachnospiraceae bacterium ND 2006 and AsCpf1 from Acidaminococcus sp. BV3L6 induce DNA modifications in human cells with equal or greater efficiency than do Cas9 orthologues, including SaCas9, StCas9, and NmCas9 from Staphylococcus aureus, Streptococcus thermophilus, and Neisseria meningitidis, respectively ${ }^{21}$. In addition, there are several reports demonstrating that LbCpfl exhibits a higher genome-wide specificity compared to SpCas $9^{22,23}$.

To test whether LbCpf1 can be used as a gene therapy tool for the treatment of retinal diseases with the advantage of a smaller gene size (3.7 kbp encoding 1,299 amino acids) compared to SpCas9, we packaged sequences encoding LbCpfl and its crRNA (total transgene cassette size; $4.7 \mathrm{kbp}$ ) in all-in-one AAV vector and delivered the resulting $\mathrm{AAV}$ into the mouse retina via intravitreal injection. We found that insertions and deletions (indels), a measure of Cpf1 activity, were induced at high frequencies in the Vegfa and Hifla genes with target specificity. In particular, Vegfa or Hifla disruption led to a long-term reduction of the area of laser-induced $\mathrm{CNV}$ without causing cone dysfunction. These findings suggest that Cpf1 has great potential as an in vivo genome editing therapy for the treatment of angiogenesis-related diseases.

\section{Results}

CRISPR-LbCpf1-mediated gene editing in vitro and in vivo. To evaluate LbCpf1-mediated genome editing of angiogenesis- associated genes, we designed several crRNAs to target Vegfa or Hif1a exons. Cpf1 nucleases complexed with these crRNAs induced indels at frequencies that ranged from $1.1 \pm 0.1 \%$ to 24.5 $\pm 0.4 \%$ in $\mathrm{C} 2 \mathrm{C} 12$ mouse myotubes (Fig. 1a; Supplementary Table 1). We selected the TS3 and TS3 crRNAs, which target the exon 1 of Vegfa and exon 8 of Hifla genes, respectively (Fig. 1b), because they both resulted in high ratio of out-of-frame indels as a representative mutation pattern with high efficiencies (Fig. 1a; Supplementary Fig. 1).

To investigate the genome editing efficiency of LbCpf1 in the mouse retina, we expressed Cpf1 with Vegfa (TS3) or Hif1a (TS3) specific crRNA in a single AAV vector plasmid (Fig. 1c). Cpf1 induced indels at frequencies of $14.5 \pm 0.7 \%$ and $12.5 \pm 0.2 \%$, respectively in $\mathrm{C} 2 \mathrm{C} 12$ mouse myotubes (Fig. 1c). Then, we packaged sequences encoding LbCpf1 and Vegfa- or Hifla-specific crRNA into an AAV serotype 9 vector. The resulting viruses were administered into the mouse eye via intravitreal injection. AAV2/9 expressing Vegfa-specific LbCpf1 (AAV-Cpf1-Vegfa) induced indels with frequencies of $57.2 \pm 4.1 \%$ and $6.5 \pm 2 \%$ in the retina and RPE, respectively, 6 weeks post-injection (Fig. 1d). Analysis of indel-bearing sequences showed that $86 \pm 0.9 \%$ and $93 \pm 2.9 \%$ carried out-of-frame mutations induced by LbCpf1 in the retina and RPE, respectively (Fig. 1d; Supplementary Fig. 2). AAV2/9 expressing Hifla-specific LbCpf1 (AAV-Cpf1-Hifla) induced indels with frequencies of $59.2 \pm 4.9 \%$ and $17.2 \pm 5.3 \%$ in the retina and RPE, respectively, 6 weeks post-injection (Fig. 1e). Analysis of indel-bearing sequences showed that $90 \pm 0.4 \%$ and 92 $\pm 0.6 \%$ carried out-of-frame mutations in the retina and RPE, respectively (Fig. 1e; Supplementary Fig. 2). As expected, an HA tag conjugated to the C-terminus of LbCpf1 was detected in both retina and RPE cells, indicating that LbCpf1 was successfully expressed (Supplementary Figs. 3, 4).

We compared indel frequencies at the Hifla target site at 4 and 6 weeks after intravitreal injection of AAV9-Cpf1-Hifla. We observed an increase in indel frequencies from $3.7 \pm 1.6 \%$ to $17.2 \pm 5.3 \%$ in RPE cells but did not observe an increase in the retina (Supplementary Fig. 5).

Target specificity of LbCpf1 in the mouse retina. We next investigated whether LbCpf1 has off-target nuclease activity in the mouse eye. To determine the genome-wide specificity of the Vegfa-or Hifla-targeting LbCpf1 nuclease, we first carried out nuclease-digested whole genome sequencing (Digenome-seq). Cell-free mouse genomic DNA was digested in vitro using the Vegfa- or Hifla-targeting LbCpf1 ribonucleoprotein (RNP) complex and then subjected to whole genome sequencing (WGS) (Fig. 2). The Vegfa- or Hifla-targeting LbCpf1 cleaved two sites or one site, respectively, including the on-target site, in the mouse genome (Fig. 2a, b; Supplementary Table 2). These results are in line with previous reports showing the high specificity of Cpf1 in the human or mouse genome $22-24$. Next, we performed targeted deep sequencing at the Digenome-seq captured off-target site using genomic DNA isolated from AAV-Cpf1-Vegfa edited mouse retina and RPE. No off-target indels were detectably induced in RPE (Fig. 2c; Supplementary Table 3). In the Cpf1 edited retina, off-target indels were detected with a frequency of $0.17 \pm 0.02 \%$. We additionally analyzed 1 potential off-target site in the mouse genome, which differed from the Vegfa or Hifla on-target site by up to four nucleotides in AAV-Cpf1-Vegfa or-Hifla treated retina and RPE. Potential sites were identified using the Cas-OFFinder program. No off-target indels were detectably induced in AAV-Cpf1-Vegfa or -Hifla injected mouse retina or RPE cells (Supplementary Fig. 6; Supplementary Table 4). Taken together, these results show that the LbCpf1 nuclease is targeted to Vegfa or Hifla in the mouse retina in a highly specific manner in vivo. 
a
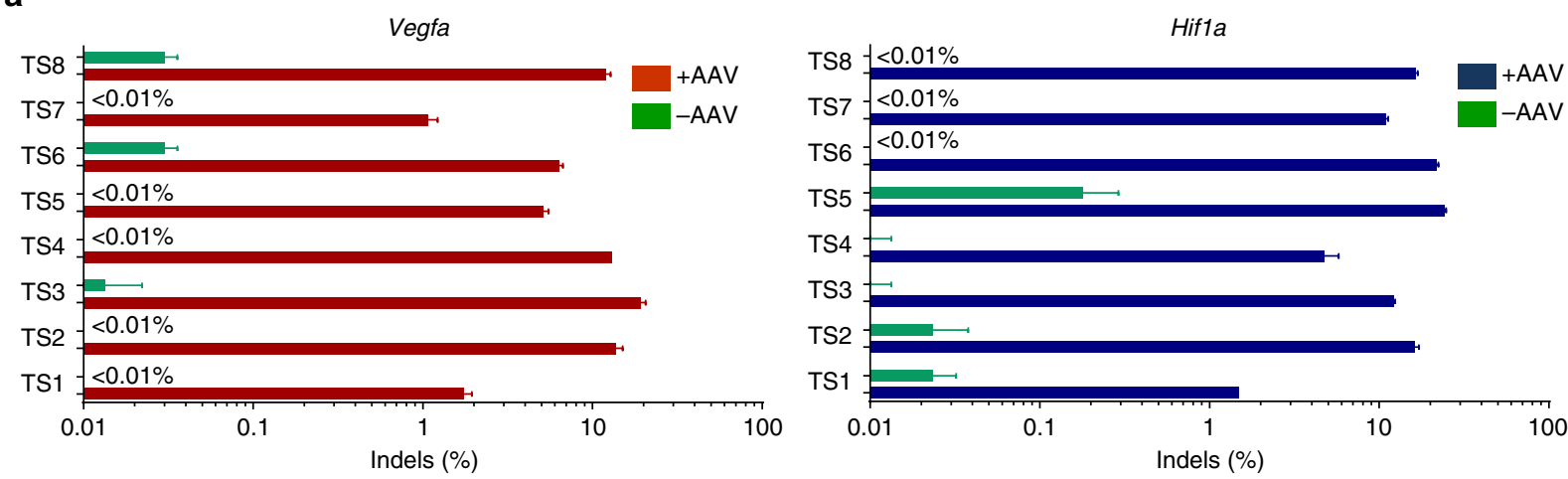

b

C
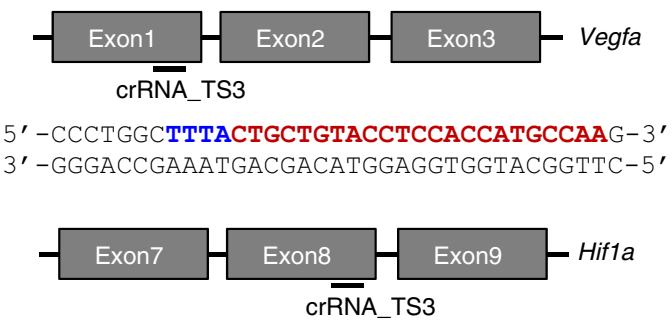

5' - CCACAGGACAGTACAGGATGCTTGCCAAAAGAGG-3' 3' -GGTGTCCTGTCATGTCCTACGAACGGTTTTCTCC-5'
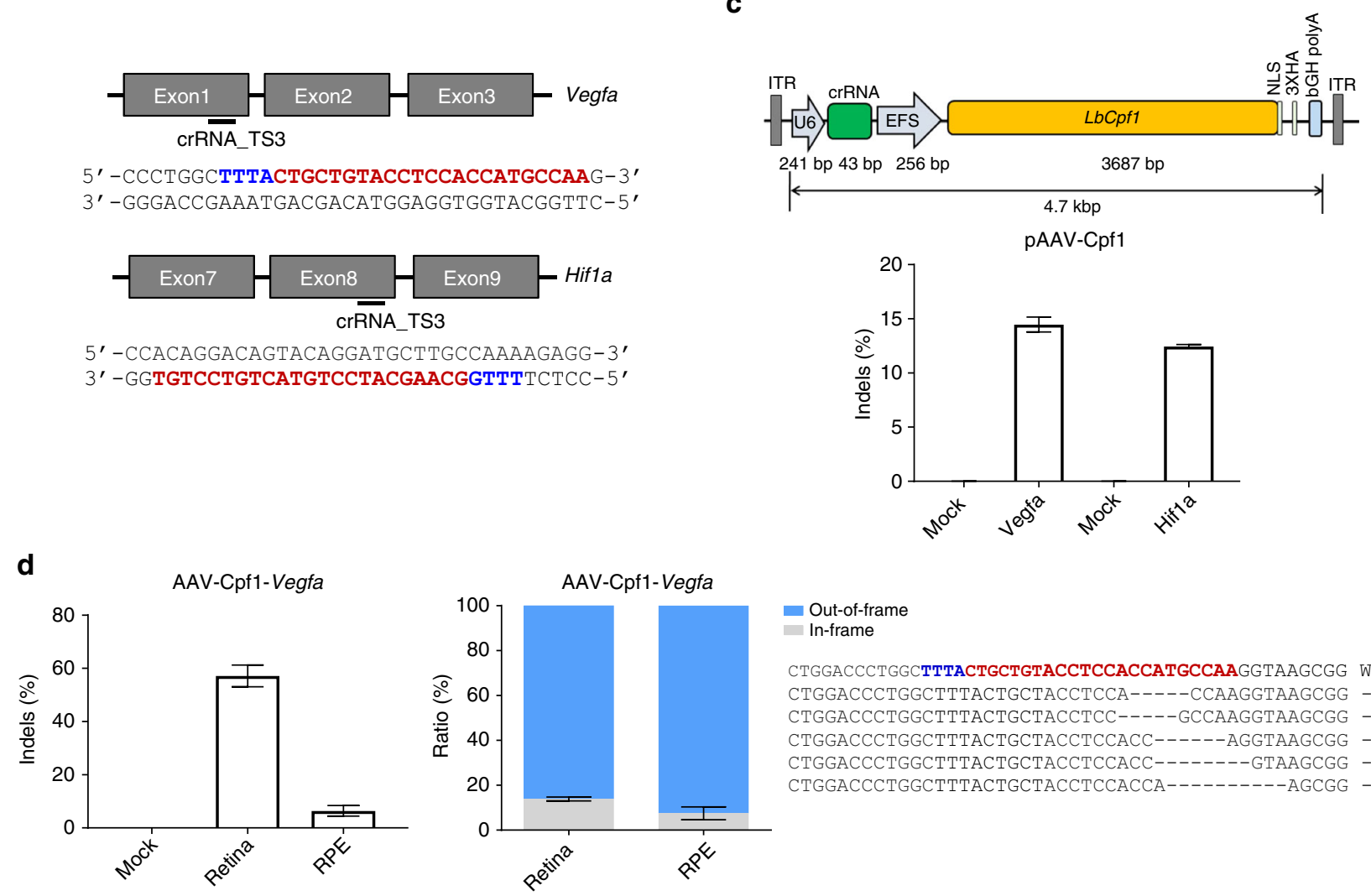

e
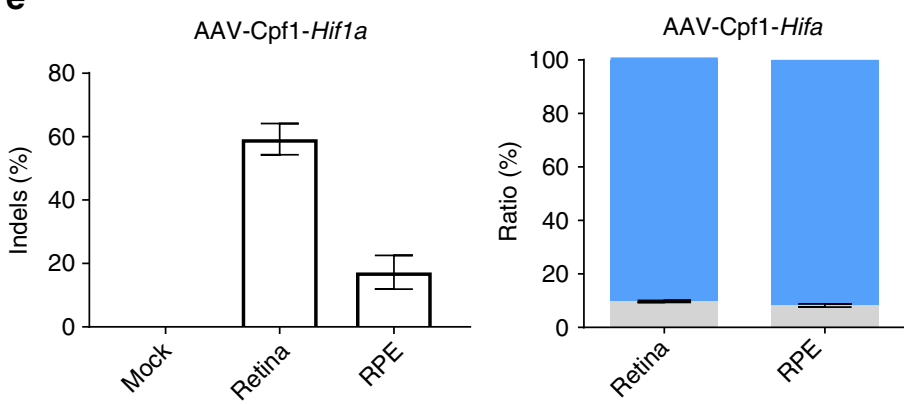

Out-of-frame

In-frame

CTGGACCCTGGCTTTACTGCTGTACCTCCACCATGCCAAGGTAAGCGG WT CTGGACCCTGGCTTTACTGCTACCTCCA----CCAAGGTAAGCGG -5 CTGGACCCTGGCTTTACTGCTACCTCC-----GCCAAGGTAAGCGG -5 CTGGACCCTGGCTTTACTGCTACCTCCACC------AGGTAAGCGG -6 CTGGACCCTGGCTTTACTGCTACCTCCACC-------GTAAGCGG -8 CTGGACCCTGGCTTTACTGCTACCTCCACCA---------AGCGG -10

Fig. 1 Genome editing with LbCpf1 in vitro and in vivo. a Mutation frequencies at the Vegfa and Hif1a target sites in C2C12 cells co-transfected with various crRNAs and LbCpf1 genome editing efficiencies were examined by deep sequencing using genome DNA isolated from cells after $48 \mathrm{~h}$ of transfection. Error bars indicate s.e.m. $(n=3)$. b The Cpf1 target sequences in the Vegfa and Hifla genes. The PAM sequence and the crRNA target sequence (TS3) are shown in blue and red, respectively. c All-in-one AAV vector plasmids encoding Cpf1 and its crRNA (TS3) and its mutation frequencies at the Vegfa and Hif1a target sites in $\mathrm{C} 2 \mathrm{C} 12$ cells transfected with pAAV-Cpf1-Vegfa or Hif1a plasmids $48 \mathrm{~h}$ post-transfection. d,e Indel frequencies and mutant sequences presented with two fractions, in-frame versus out-of-frame indels at the Vegfa (d) or Hifla (e) target site in the retina and RPE cells using deep sequencing 6 weeks post-intravitreal injection of AAV-Cpf1-Vegfa or AAV-Cpf1-Hif1a. The PAM sequence and the crRNA target sequence (TS3) are shown in blue and red, respectively. Error bars indicate s.e.m. $(n=4)$ 
a

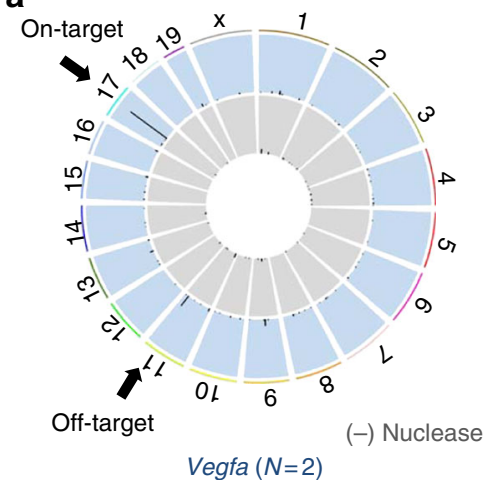

b

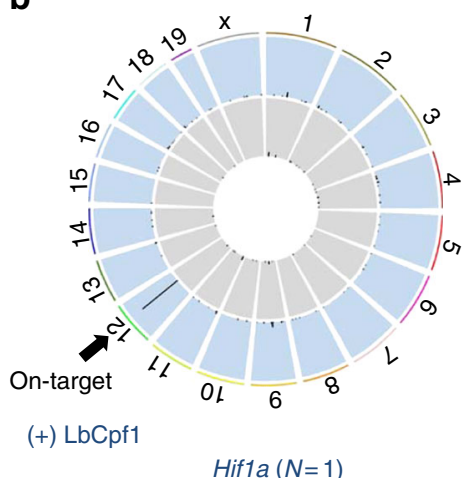

C

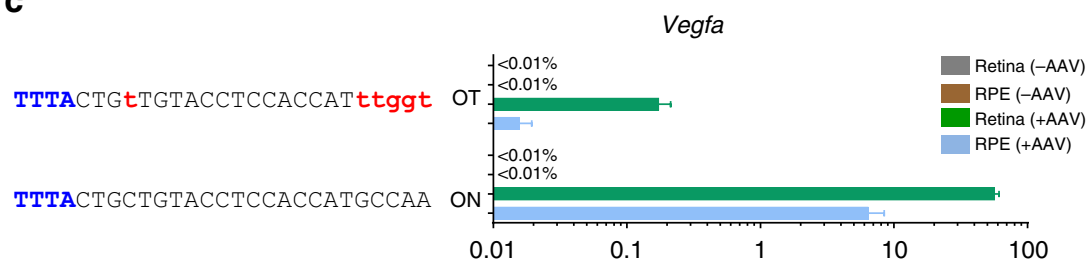

Fig. 2 Retinal genome editing specificity of LbCpf1. a,b Mouse genomic DNA isolated from C57BL/6 J mice was digested in vitro by LbCpf1 and its crRNA targeted to Vegfa (a) or Hif1a (b) and subjected to whole-genome sequencing. Circos plots show genome-wide DNA cleavage scores across the mouse genome. Arrows indicate on-target or off-target sites. $N$ indicates the number of in vitro cleavage sites identified by Digenome-seq. $\mathbf{c}$ Indel frequencies at in vitro cleavage sites identified by Digenome-seq. Genomic DNA isolated from retina and RPE cells 6 weeks after injection of AAV-Cpf1-Vegfa was subjected to targeted deep sequencing. Mismatched nucleotides are shown in red and PAM sequences in blue. ON, on-target site; OT, off-target site. Error bar indicates s.e.m. $(n=4)$

Therapeutic genome editing for the treatment of CNV. Next, we induced $\mathrm{CNV}$ in the eye by laser treatment 6 weeks after injection of AAV-Cpf1-Vegfa or -Hifla and measured the area of $\mathrm{CNV}^{25} 1$ week later (Fig. 3a). AAV-Cpf1-Vegfa or -Hifla reduced the area of CNV by $42 \pm 4 \%$ and $34 \pm 5 \%$, respectively, compared to the AAV-uninjected negative control (Fig. 3b). A DNMT-specific $\mathrm{Cpf1}^{22}$, used as another negative control, which expresses crRNA but does not induce double strand breaks, also did not show any therapeutic effect. Additionally, we found that AAVCpf1-Vegfa or -Hifla reduced VEGFA protein levels in the RPE by $17 \pm 3 \mathrm{pg} / \mathrm{mg}$ and $15 \pm 2 \mathrm{pg} / \mathrm{mg}$, respectively, compared to the AAV-uninjected negative control (Fig. 3c). In contrast, the VEGFA protein levels in the retina were not significantly different among groups (Fig. 3c). These data imply that the decrease in the level of VEGFAprotein in the RPE might be linked to the decrease in the CNV area. When aflibercept, a widely-used anti-VEGF drug, was injected the same day as the laser treatment, it reduced the area of $\mathrm{CNV}$ by $39 \pm 6 \%$ relative to the $\mathrm{AAV}$-uninjected negative control, comparable to the therapeutic effect observed with injection of AAV-Cpf1-Vegfa or -Hifla (Fig. 3a, b). However, when aflibercept was injected 6 weeks before the laser treatment, the area of CNV was not reduced (Fig. 3d, e), in line with the short half-life of the drug ${ }^{26}$. This result suggests that genome editing has a long-term therapeutic effect, whereas aflibercept must be injected multiple times to maintain its therapeutic effect on CNV.

No retinal dysfunction after injection of AAV-Cpf1-Vegfa or -Hif1a. The conditional knockout of the Vegfa gene in mouse RPE cells leads to cone dysfunction ${ }^{27}$. To evaluate the potential toxicity of AAV-Cpf1-mediated Vegfa or Hifla gene disruption, we first performed full-field electroretinography (ERG) in mice at 6 weeks after the intravitreal injection of AAV-Cpf1-Vegfa or -Hifla. We observed no significant decrease in the scotopic response in these mice compared to that in untreated mice (Fig. 4a, b; Supplementary Fig. 7). In addition, there was no significant change in the photopic response (Fig. 4c; Supplementary Fig. 7). Then, we evaluated the level of opsin expression in the retina at 6 months after the intravitreal injection of AAVCpf1-Vegfa or-Hif1a. We also found that AAV-Cpf1-Vegfa or -Hifla did not affect the size of the opsin-positive area in the retina, which is closely related to cone function (Fig. 4d, e), suggesting that AAV-Cpfl-mediated Vegfa or Hifla gene disruption provides a safe therapeutic window.

\section{Discussion}

In this report, we have shown that LbCpf1 can be successfully delivered to the mouse retina via an AAV serotype 9 vector and that it can induce efficient gene editing in the retina and RPE cells. LbCpf1 induced indels at high frequencies in the Vegfa and Hifla genes, suggesting its potential for the treatment of angiogenesisassociated diseases. Cpf1 has several benefits for therapeutic applications. First, Cpf1 is highly specific in human cells with minimum off-target activities, compared to SpCas9 as shown in previous reports 22,23 . The use of Cpf1 also allows for streamlined multiplex genome editing. Because Cpf1 can process pre-crRNA arrays, multiple pre-crRNAs can be expressed from a single U6 promoter without the need for additional sequences ${ }^{19,28}$.

In vivo therapeutic genome editing is an active area of research. However, unlike ex vivo approaches, in vivo genome editing is limited by potential immune responses against Cas 9 or Cpf1 and relatively poor efficiency of transgene delivery. In this regard, the eye is an ideal organ for in vivo therapeutic editing because it is immune-privileged. AAV vectors are powerful tools for delivering the genome engineering machinery to the ocular tissues $29-31$. Indeed, a number of studies have demonstrated efficient expression of transgenes using AAV serotype 9 in the mouse retina ${ }^{32-35}$. In our previous study, we also showed that Cas9 derived from Campylobacter jejuni is efficiently expressed in the retina and RPE cells after intravitreal injection via an AAV9 vector ${ }^{35}$. In this study, we used AAV serotype 9 as a vehicle to deliver Cpf1 and 
No AAV

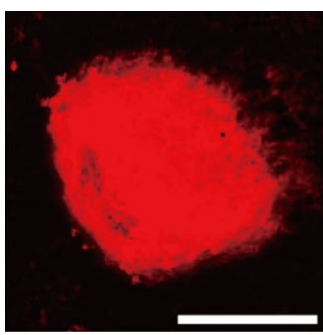

b

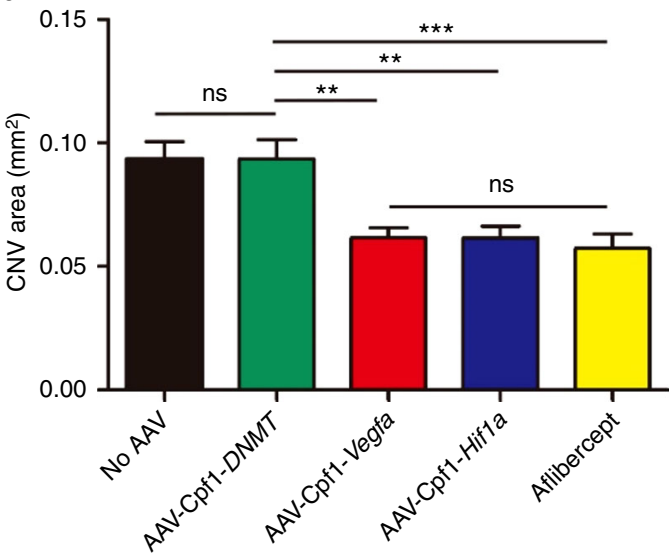

AAV-Cpf1: Vegfa

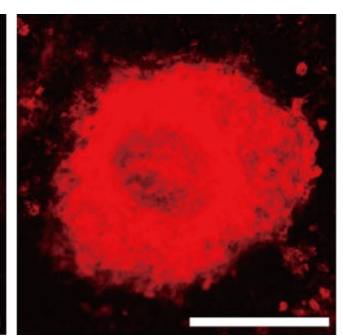

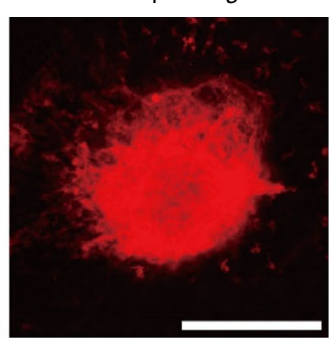

C
AAV-Cpf1: Hif1a

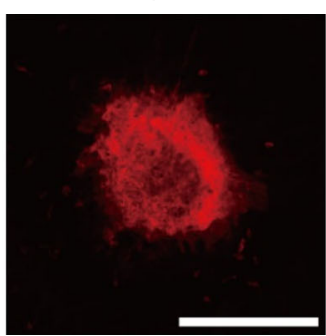

Aflibercept

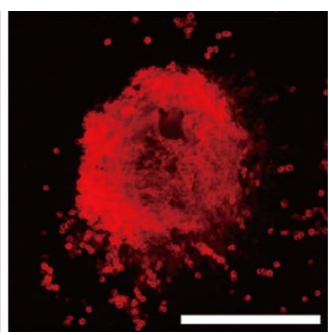

Retina in CNV
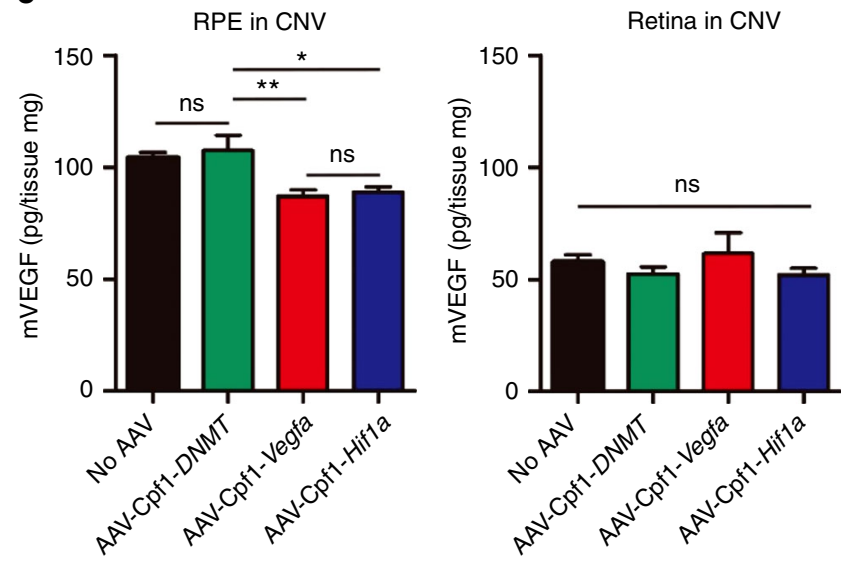

d

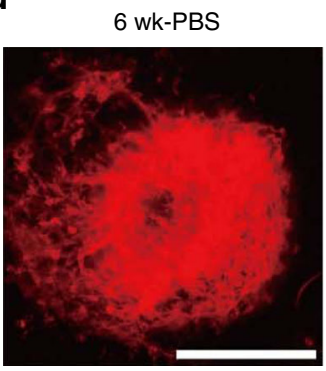

6 wk-Aflibercept

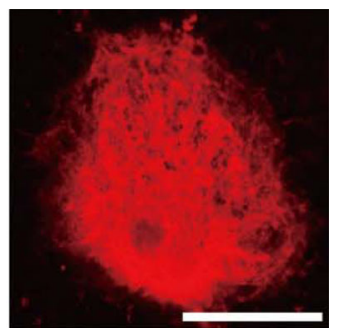

e

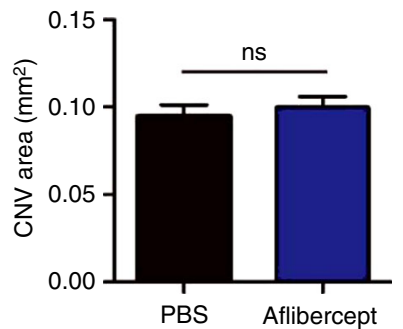

Fig. 3 LbCpf1 targeted to Vegfa or Hifla reduces the area of laser-induced CNV in mice. a-c At day 42 post-injection of AAV-Cpf1, mice were treated with laser to induce CNV. Aflibercept $(2.5 \mu \mathrm{g})$ was intravitreally injected immediately after laser treatment. No AAV indicates no injection of AAV or aflibercept as a control. Three days after laser treatment, Vegfa protein levels were measured by ELISA in the retina and RPE complex. One week after laser treatment, the CNV area was analyzed. a Representative laser-induced CNV stained with isolectin B4 in mouse eyes injected with AAV-Cpf1 targeted to DNMT, Vegfa, or Hif1a. Scale bar is $200 \mu \mathrm{m}$. b The CNV area. Error bars indicate s.e.m. $(n=20)$. One-way ANOVA and Tukey's post-hoc tests, ${ }^{\star} P<0.05,{ }^{\star \star} P<0.01$, ${ }^{\star \star \star} P<0.001$, ns, not significant. c VEGFA protein levels. Error bars indicate s.e.m. $(n=10)$. One-way ANOVA and Tukey's post-hoc tests. ${ }^{\star}, P<0.05$; $\star \star, P<0.01$; ns not significant. $\mathbf{d}$-e Six weeks after intravitreal injection of PBS or aflibercept $(2.5 \mu \mathrm{g})$, mice were treated with laser to induce CNV. One week after laser treatment, the CNV area was analyzed. $\mathbf{d}$ Representative laser-induced CNV stained with isolectin B4 in mouse eyes injected with PBS or aflibercept. Scale bar is $200 \mu \mathrm{m}$. e The CNV area. Error bars indicate s.e.m. $(n=30)$. Student's $t$-test. ns, not significant

crRNA into mouse retina. In contrast to AAV serotype 2, which targets RPE cells only when delivered by subretinal injection, AAV9 was efficiently transfected into RPE cells after intravitreal injection $^{35}$.

AAV-mediated genome editing may cause systemic side effects if AAV passes through the bloodstream during intravitreal injection. However, several reports support the safety of AAV in the treatment of retinal diseases. In LCA clinical trials, there was no evidence of systemic dissemination of AAV vectors in tears, serum, or saliva samples 30 days after subretinal administration of AAV2/2-hRPE65 $5^{36-38}$. Sugano et al. and Shih et al. also assessed the presence of AAV following intravitreal injection: AAV was detected within the ocular tissues but not in other organs ${ }^{39,40}$. However, a study of the immune response to Cpf1 should also be investigated.
When delivered to the retina and RPE, AAV-Cpf1-Vegfa or Hifla induced Vegfa or Hifla gene disruption, respectively followed by reduction of the CNV area in a mouse model of AMD. Its anti-angiogenic effect was comparable to that of aflibercept. Note, however, that unlike AAV-Cpf1-Vegfa or Hif1a, aflibercept did not inhibit neovascularization and did not reduce the area of $\mathrm{CNV}$ when given 6 weeks before the laser treatment, confirming its short half-life in vivo ${ }^{26}$. This finding suggests that AAV-LbCpf1 has a long-term therapeutic effect even with a single administration and could replace anti-VEGF drugs currently in use in the clinic. Note that aflibercept and other anti-VEGF drugs must be administered repetitively, often for a lifetime. Evidence of long-term expression of sequences delivered by AAV is supported by several promising, ongoing clinical trials using AAV vectors for retinal disorders ${ }^{41}$. It has also been 
a

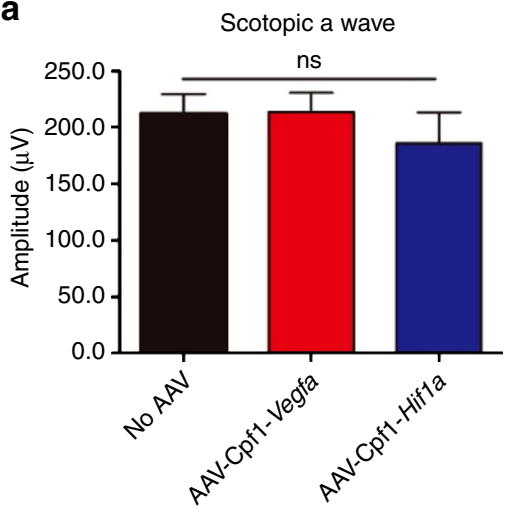

C

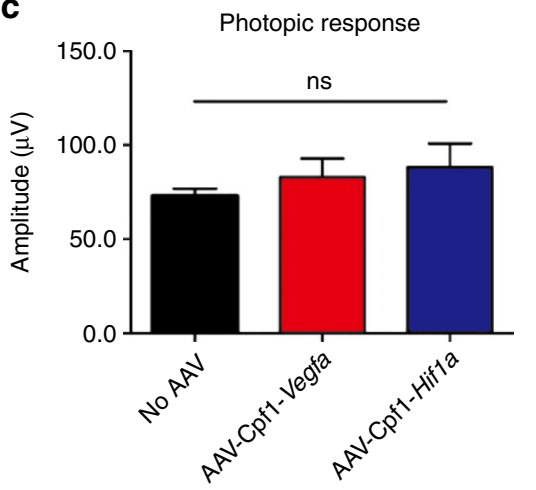

b

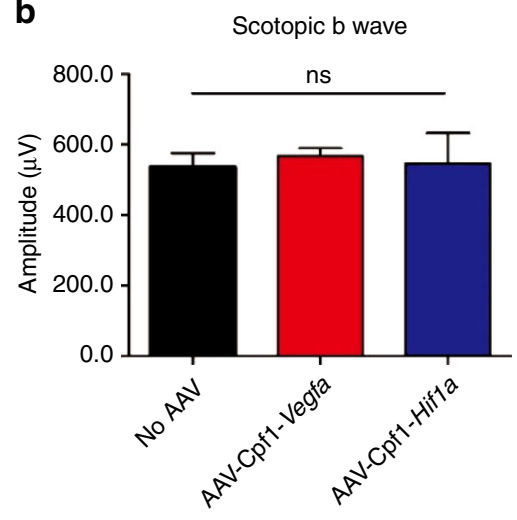

d

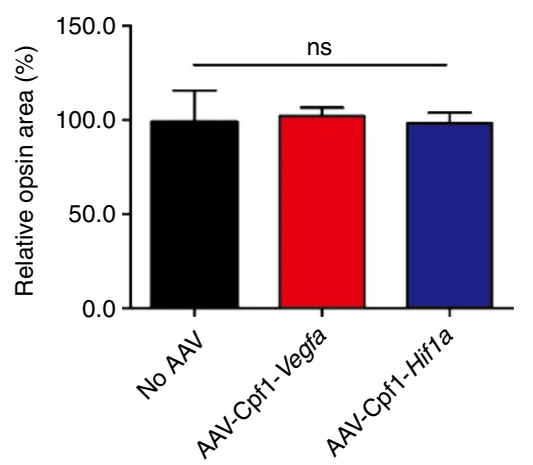

$\mathbf{e}$

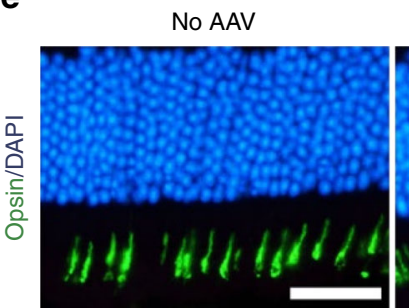

AAV-Cpf1: Vegfa

AAV-Cpf1: Hif1a
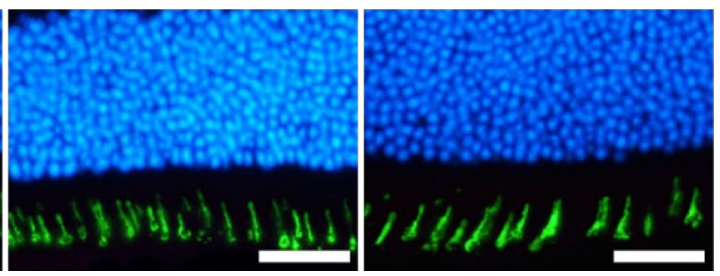

Fig. 4 LbCpf1 targeted to Vegfa or Hif1a does not affect cone function. a-c At day 42 post-AAV injection, full-field ERG was performed to evaluate retinal function. There was no significant decrease in the a scotopic a wave, $\mathbf{b}$ scotopic b wave, or c photopic response in mice treated with AAV-Cpf1-Vegfa or -Hifla compared to normal control mice. Error bars indicate s.e.m. $(n=4)$. One-way ANOVA. ns, not significant. d, e Opsin-positive areas in the retina at day 42 post-injection. $\mathbf{d}$ Relative opsin-positive areas of the AAV-Cpf1-injected mice were normalized to that of the AAV-uninjected negative control mice. Error bars indicate s.e.m. $(n=4)$. Student's $t$-test. ns not significant. e Representative images of opsin positive areas in mice treated with AAV-Cpf1-Vegfa or -Hifla compared to the AAV-uninjected negative control mice (which received no AAV). Scale bar $20 \mu \mathrm{m}$

reported that $\mathrm{AAV}$-mediated transgene expression continued in non-human primates for as long as 5.5 years after gene transfer without any detectable toxicity ${ }^{42}$. The ability of AAV vectors to mediate long-term transgene expression offers a means of treating life-long chronic diseases such as AMD with a single administration of vector. As such, the use of AAV-Cpf1 may reduce the cost burden of treatment for patients and improve the quality of life. Our results support that application of AAV-LbCpf1 as a gene editing tool will be an effective strategy for treating AMD and other diseases associated with neovascularization.

\section{Methods}

Construction of an AAV vector plasmid encoding LbCpf1 and crRNA. A human codon-optimized LbCpf1 coding sequence, derived from Lachnospiraceae bacterium ND 2006, was purchased from Addgene (plasmid \# 78744). The sequence was cloned into an AAV inverted terminal repeat (ITR)-based vector plasmid. The Vegfa, HIf1a, or DNMT targeting crRNA sequence (Supplementary Table 1) was also inserted to create pAAV-ITR-LbCpf1-crRNA. crRNAs were transcribed under the control of the U6 promoter and LbCpf1 expression was controlled by the elongation factor-1 alpha short (EFS) promoter in C2C12 (ATCC, CRL-1772) cells and in the mouse retina and RPE.

Cell culture and mutation analysis. Cells were maintained in Dulbecco's Modified Eagle's Medium (DMEM, Welgene, cat. no. LM001-05) supplemented with 100 units per $\mathrm{ml}$ penicillin (Gibco, cat. no. 15140-122), $100 \mu \mathrm{g} / \mathrm{ml}$ streptomycin, and $10 \%$ fetal bovine serum heat-inactivated (FBS, Welgene, cat. no. S 101-01). In this study, we investigated the efficacy of several crRNAs in $\mathrm{C} 2 \mathrm{C} 12$ cells because these cells are efficiently transfected. Cells $\left(1 \times 10^{5}\right)$ were seeded into 24 -well plates one day prior to transfection and transfected with the crRNA plasmid (1500 ng) and the Cpf1 plasmid (500 ng) using $4 \mu \mathrm{l}$ of Lipofectamine 2000 (Invitrogen, cat. no. 11668019). Cells were maintained in DMEM supplemented with 2\% FBS for differentiation. Genomic DNA was isolated using a DNeasy Blood \& Tissue kit (Qiagen, cat. no. 69581) $48 \mathrm{~h}$ post-transfection. On-target or off-target loci were amplified using $100 \mathrm{ng}$ of genomic DNA for targeted deep sequencing. Deepsequencing libraries were generated by PCR. TruSeq HT Dual Index primers were used to label each sample. Pooled libraries were subjected to paired-end sequencing using MiniSeq (Illumina). Indel frequencies are described in Supplementary Table 1.

Production and titration of AAV vectors. To produce AAV vectors, they were pseudotyped in AAV9 capsids. HEK293T cells (ATCC, CRL-3216) were transfected with pAAV-ITR-LbCpf1-crRNA, pAAV2/9 encoding for AAV2rep and 
AAV9cap, and helper plasmid. HEK293T cells were cultured in DMEM with 2\% FBS. Recombinant pseudotyped AAV vector stocks were generated using PEI coprecipitation with PEIpro (Polyplus-transfection) and triple-transfection with plasmids at a molar ratio of 1:1:1 in HEK293T cells. After $72 \mathrm{~h}$ of incubation, cells were lysed and particles were purified by iodixanol (Sigma-Aldrich) step-gradient ultracentrifugation. The number of vector genomes was determined by quantitative PCR.

Digenome sequencing. Genomic DNA was isolated from liver tissue of C57BL/6 mice using a DNeasy Blood \& Tissue kit (Qiagen) according to the manufacturer's instructions. Genomic DNA $(8 \mu \mathrm{g})$ was mixed with LbCpf1 protein $(300 \mathrm{nM})$ and crRNA $(900 \mathrm{nM})$ in a $400 \mu$ l reaction buffer $(100 \mathrm{mM} \mathrm{NaCl}, 50 \mathrm{mM}$ Tris- $\mathrm{HCl}, 10$ $\mathrm{mM} \mathrm{MgCl} 2$, and $100 \mu \mathrm{g} / \mathrm{ml} \mathrm{BSA}$ ) and the mixture was incubated for $8 \mathrm{~h}$ at $37^{\circ} \mathrm{C}$. Digested genomic DNA was then incubated with RNase A $(50 \mu \mathrm{g} / \mathrm{ml})$ for $30 \mathrm{~min}$ at $37^{\circ} \mathrm{C}$ to degrade crRNAs and purified again with a DNeasy Blood \& Tissue kit (Qiagen). Digested DNA was fragmented using the Covaris system and ligated with adapters for library formation. DNA libraries were subjected to whole-genome sequencing using an Illumina HiSeq X Ten Sequencer at Macrogen (South Korea) 43,44. We used the Isaac aligner to generate a Bam file using the following parameters: ver. 01.14.03.12; Mouse genome reference, mm10 from UCSC; Base quality cutoff, 15; Keep duplicate reads, yes; Variable read length support, yes; Realign gaps, no; and Adapter clipping, yes (adapter: AGATCGGAAGAGC ${ }^{*}$, ${ }^{*}$ GCTCTTCCGATCT) ${ }^{45}$. A DNA cleavage score was assigned to each nucleotide position across the entire genome, using WGS data, according to the equation presented in Kim et $\mathrm{a}^{22}$. These equations assume that Cpf1 produces 5' 1- to 5-nt overhangs. In vitro cleavage sites with DNA cleavage scores above the cut-off value of 2.5 were computationally identified.

Animals. The care, use, and treatment of all animals in this study were in strict agreement with the ARVO statement for the Use of Animals in Ophthalmic and Vision Research, the College of Veterinary Medicine guidelines, and the guidelines established by the Seoul National University Institutional Animal Care and Use Committee, which granted permission to perform animal experiments. Eightweek-old, male, specific pathogen-free C57BL/6 J mice $(n=4-9)$ were used in this study. Mice were maintained under a $12 \mathrm{~h}$ dark-light cycle.

Laser-induced CNV model. After mice were anesthetized, pupils were dilated with an eye drop containing phenylephrine $(0.5 \%)$ and tropicamide $(0.5 \%)$. Laser photocoagulation was performed using an indirect head set delivery system (Iridex) and laser system (Ilooda). Laser parameters were $810 \mathrm{~nm}$ wave length, $200 \mu \mathrm{m}$ spot size, $800 \mathrm{~mW}$ power, and $70 \mathrm{~ms}$ exposure time. Laser burn was induced 3-4 times around the optic disc. Only burns that produced a bubble without vitreous hemorrhage were included in the study. Seven days later, the eyes were fixed in $4 \%$ paraformaldehyde for $1 \mathrm{~h}$ at room temperature. RPE complexes (RPE/choroid/ sclera) were prepared for immunostaining and then incubated with isolectin-B4 (Thermo Fisher Scientific, cat. no. I21413, 1:100) overnight at $4{ }^{\circ} \mathrm{C}$. The RPE complex was flat-mounted and viewed with a fluorescent microscope (Eclipse 90i, Nikon) or a confocal microscope (LSM 710, Carl Zeiss) at a magnification of 100x. The CNV area was measured using Image J software $(1.47 \mathrm{v}, \mathrm{NIH})$ by blinded observers. An average of 3-4 CNV areas per eye were analyzed.

Intravitreal injection of AAV. Eight-week-old mice were anesthetized with an intraperitoneal injection of a mixture of tiletamine and zolazepam $(1: 1,2.25 \mathrm{mg} / \mathrm{kg}$ body weight) and xylazine hydrochloride $(0.7 \mathrm{mg} / \mathrm{kg}$ body weight). AAV2/9LbCpf1-Vegfa or -Hifla $\left(2 \times 10^{10}\right.$ viral genomes in $\left.2 \mu \mathrm{l}\right)$ was intravitreally injected using a Nanofil syringe with a $33 \mathrm{G}$ blunt needle (World Precision Instruments Inc.) under an operating microscope (Leica Microsystems Ltd.). The virus dose used in this study was limited due to production issues, and higher virus doses may elicit higher mutation efficacy.

Immunofluorescent staining and imaging of retinal tissue. For the analysis of the opsin-positive area, formalin-fixed paraffin embedded samples were prepared at day 42 post-injection $(n=4)$. Cross-section samples were immunostained with anti-opsin antibody (Millipore, cat. no. AB5405, 1:1000) and Alexa Fluor 488 antibody (Thermo Fisher Scientific, cat. no. A-11034, 1:500). The opsin-positive area was measured using Image J software $(1.47 \mathrm{v}, \mathrm{NIH})$ by blinded observers. To visualize the distribution of HA-tagged Cpfl, the eyes were fixed in $4 \%$ paraformaldehyde for $1 \mathrm{~h}$ at room temperature. RPE complexes (RPE/choroid/sclera) were prepared for immunostaining and then incubated with anti-HA antibody (Roche, cat. no. 3F10, 1:100) overnight at $4{ }^{\circ} \mathrm{C}$. After staining with Alexa Fluor 594 antibodies (Thermo Fisher Scientific, cat. no. A-11006, 1:500), the RPE flat-mounts were imaged using a confocal microscope (LSM 710, Carl Zeiss). The scanning parameters were as follows: scaling $(x=0.042 \mu \mathrm{m} /$ pixel, $y=0.042 \mu \mathrm{m} /$ pixel, $z=$ $0.603 \mu \mathrm{m} / \mathrm{pixel}$ ), dimensions ( $x=1024, y=1024$, channels: 2 , 8-bit) with objective C-Apochromat $40 \times / 1.20 \mathrm{~W}$ Korr M27. ZEN 2 software was used to process the images.
Mouse VEGFA ELISA. At day 42 post-injection of AAV2/9-LbCpf1, mice were treated with laser. Three days after laser treatment, whole RPE complexes were separated from neural retina tissue and frozen for further analysis. Sample tissues were lysed in RIPA buffer $(120 \mu \mathrm{l})$ and Vegfa protein levels were measured using a mouse VEGF Quantikine ELISA kit (R\&D systems, cat. no. MMV00) according to the manufacturer's instructions.

Electroretinography (ERG) analysis. Mice were dark-adapted over $16 \mathrm{~h}$. Mice were anesthetized with an intraperitoneal injection of a mixture of tiletamine and zolazepam (1:1, $2.25 \mathrm{mg} / \mathrm{kg}$ body weight) and xylazine hydrochloride $(0.7 \mathrm{mg} / \mathrm{kg}$ body weight). Pupils were dilated with an eye drop containing phenylephrine $(0.5 \%)$ and tropicamide $(0.5 \%)$. Contact lens electrodes were placed on both eyes with a drop of methylcellulose. Full-field ERGs were recorded ${ }^{46}$ by using the universal testing and electrophysiologic system 2000 (UTAS E-2000, LKC Technologies). The responses were recorded at a gain of $2 \mathrm{k}$ using a notch filter at $60 \mathrm{~Hz}$, and were bandpass filtered between 0.1 and $1500 \mathrm{~Hz}$. In the light-adapted photopic state, with a $30 \mathrm{~cd} / \mathrm{m}^{2}$ background light to desensitize the rods and isolate cones, photopic cone responses were recorded in response to a single flash of $0 \mathrm{~dB}$. The amplitude of the a-wave was measured from the baseline to the lowest negativegoing voltage, whereas peak b-wave amplitudes were measured from the trough of the a-wave to the highest peak of the positive b-wave.

Statistical analysis. No statistical methods were used to predetermine sample size for in vitro or in vivo experiments. All group results are expressed as mean \pm SEM, if not stated otherwise. Comparisons between groups were made using the twotailed Student's $t$-test or one-way ANOVA and Tukey post-hoc tests for multiple groups. Statistical significance as compared to untreated controls is denoted with * $(P<0.05),{ }^{* *}(P<0.01),{ }^{* *}(P<0.001)$ in the figures and figure legends. Statistical analysis was performed in Graph Pad PRISM 5.

Data availability. The deep sequencing data from this study have been submitted to the NCBI Sequence Read Archive under accession number SRP129908. The data that support the findings of this study are available from the corresponding author upon reasonable request.

Received: 17 October 2017 Accepted: 6 April 2018

Published online: 10 May 2018

\section{References}

1. Cheung, L. K. \& Eaton, A. Age-related macular degeneration. Pharmacotherapy 33, 838-855 (2013).

2. Ferrara, N. Vascular endothelial growth factor and age-related macular degeneration: from basic science to therapy. Nat. Med. 16, 1107-1111 (2010).

3. Amoaku, W. M. et al. Defining response to anti-VEGF therapies in neovascular AMD. Eye 29, 1397-1398 (2015).

4. Hanout, M. et al. Therapies for neovascular age-related macular degeneration: current approaches and pharmacologic agents in development. Biomed. Res. Int. 2013, 830837 (2013).

5. Funk, M. et al. Neovascular age-related macular degeneration: intraocular cytokines and growth factors and the influence of therapy with ranibizumab. Ophthalmology 116, 2393-2399 (2009).

6. Sawada, O. et al. Aqueous vascular endothelial growth factor after intravitreal injection of pegaptanib or ranibizumab in patients with age-related macular degeneration. Retina 30, 1034-1038 (2010).

7. Yiu, G., Tieu, E., Nguyen, A. T., Wong, B. \& Smit-McBride, Z. Genomic disruption of VEGF-A expression in human retinal pigment epithelial cells using CRISPR-Cas9 endonuclease. Invest. Ophthalmol. Vis. Sci. 57, 5490-5497 (2016).

8. Barrangou, R. et al. CRISPR provides acquired resistance against viruses in prokaryotes. Science 315, 1709-1712 (2007).

9. Cong, L. et al. Multiplex genome engineering using CRISPR/Cas systems. Science 339, 819-823 (2013)

10. Mali, P. et al. RNA-guided human genome engineering via Cas9. Science 339 823-826 (2013).

11. Cho, S. W., Kim, S., Kim, J. M. \& Kim, J. S. Targeted genome engineering in human cells with the Cas9 RNA-guided endonuclease. Nat. Biotechnol. 31, 230-232 (2013).

12. Jinek, M. et al. RNA-programmed genome editing in human cells. eLife 2, e00471 (2013)

13. Koo, T. \& Kim, J. S. Therapeutic applications of CRISPR RNA-guided genome editing. Brief. Funct. Genom. 16, 38-45 (2017)

14. Dai, W. J. et al. CRISPR-Cas9 for in vivo gene therapy: promise and hurdles. Mol. Ther. Nucl. Acids 5, e349 (2016). 
15. Fellmann, C., Gowen, B. G., Lin, P. C., Doudna, J. A. \& Corn, J. E. Cornerstones of CRISPR-Cas in drug discovery and therapy. Nat. Rev. Drug. Discov. 16, 89-100 (2017).

16. Sanchez-Rivera, F. J. \& Jacks, T. Applications of the CRISPR-Cas 9 system in cancer biology. Nat. Rev. Cancer 15, 387-395 (2015).

17. Jinek, M. et al. A programmable dual-RNA-guided DNA endonuclease in adaptive bacterial immunity. Science 337, 816-821 (2012).

18. Zetsche, B. et al. Cpf1 is a single RNA-guided endonuclease of a class 2 CRISPR-Cas system. Cell 163, 759-771 (2015).

19. Fonfara, I., Richter, H., Bratovic, M., Le Rhun, A. \& Charpentier, E. The CRISPR-associated DNA-cleaving enzyme Cpf1 also processes precursor CRISPR RNA. Nature 532, 517-521 (2016).

20. Kim, H. K. et al. In vivo high-throughput profiling of CRISPR-Cpf1 activity. Nat. Methods 14, 153-159 (2017).

21. Toth, E. et al. Cpf1 nucleases demonstrate robust activity to induce DNA modification by exploiting homology directed repair pathways in mammalian cells. Biol. Direct 11, 46 (2016).

22. Kim, D. et al. Genome-wide analysis reveals specificities of Cpf1 endonucleases in human cells. Nat. Biotechnol. 34, 863-868 (2016).

23. Kleinstiver, B. P. et al. Genome-wide specificities of CRISPR-Cas Cpf1 nucleases in human cells. Nat. Biotechnol. 34, 869-874 (2016).

24. Hur, J. K. et al. Targeted mutagenesis in mice by electroporation of Cpf1 ribonucleoproteins. Nat. Biotechnol. 34, 807-808 (2016).

25. Weinberger, D. et al. Mobile laser indirect ophthalmoscope: for the induction of choroidal neovascularization in a mouse model. Curr. Eye. Res. 42, 1545-1551 (2017).

26. Park, S. J. et al. Intraocular pharmacokinetics of intravitreal aflibercept (Eylea) in a rabbit model. Invest. Ophthalmol. Vis. Sci. 57, 2612-2617 (2016)

27. Kurihara, T., Westenskow, P. D., Bravo, S., Aguilar, E. \& Friedlander, M. Targeted deletion of Vegfa in adult mice induces vision loss. J. Clin. Invest. 122, 4213-4217 (2012).

28. Zetsche, B. et al. Multiplex gene editing by CRISPR-Cpf1 using a single crRNA array. Nat. Biotechnol. 35, 31-34 (2017).

29. Huang, X. et al. Genome editing abrogates angiogenesis in vivo. Nat. Commun. 8, 112 (2017).

30. Hung, S. S. et al. AAV-mediated CRISPR/Cas gene editing of retinal cells in vivo. Invest. Ophthalmol. Vis. Sci. 57, 3470-3476 (2016).

31. Yu, W. et al. Nrl knockdown by AAV-delivered CRISPR/Cas9 prevents retinal degeneration in mice. Nat. Commun. 8, 14716 (2017).

32. Lei, B., Zhang, K., Yue, Y., Ghosh, A. \& Duan, D. Adeno-associated virus serotype-9 efficiently transduces the retinal outer plexiform layer. Mol. Vis. 15, 1374-1382 (2009).

33. Byrne, L. C., Lin, Y. J., Lee, T., Schaffer, D. V. \& Flannery, J. G. The expression pattern of systemically injected AAV9 in the developing mouse retina is determined by age. Mol. Ther. 23, 290-296 (2015).

34. Bemelmans, A. P. et al. A single intravenous AAV9 injection mediates bilateral gene transfer to the adult mouse retina. PLoS ONE 8, e61618 (2013).

35. Kim, E. et al. In vivo genome editing with a small Cas 9 orthologue derived from Campylobacter jejuni. Nat. Commun. 8, 14500 (2017)

36. Bainbridge, J. W. et al. Effect of gene therapy on visual function in Leber's congenital amaurosis. N. Engl. J. Med. 358, 2231-2239 (2008).

37. Maguire, A. M. et al. Safety and efficacy of gene transfer for Leber's congenital amaurosis. N. Engl. J. Med. 358, 2240-2248 (2008)

38. Hauswirth, W. W. et al. Treatment of leber congenital amaurosis due to RPE65 mutations by ocular subretinal injection of adeno-associated virus gene vector: short-term results of a phase I trial. Hum. Gene. Ther. 19, 979-990 (2008).

39. Sugano, E. et al. Local and systemic responses following intravitreous injection of AAV2-encoded modified Volvox channelrhodopsin-1 in a genetically blind rat model. Gene. Ther. 23, 158-166 (2016).

40. Shih, C. S. et al. AAV-mediated local delivery of interferon-beta for the treatment of retinoblastoma in preclinical models. Neuromol. Med. 11, 43-52 (2009).
41. Ochakovski, G. A., Bartz-Schmidt, K. U. \& Fischer, M. D. Retinal gene therapy: surgical vector delivery in the translation to clinical trials. Front. Neurosci. 11, 174 (2017).

42. Nathwani, A. C. et al. Long-term safety and efficacy following systemic administration of a self-complementary AAV vector encoding human FIX pseudotyped with serotype 5 and 8 capsid proteins. Mol. Ther. 19, 876-885 (2011).

43. Kim, D. et al. Digenome-seq: genome-wide profiling of CRISPR-Cas9 offtarget effects in human cells. Nat. Methods 12, 237-243 (2015).

44. Kim, D., Kim, S., Kim, S., Park, J. \& Kim, J. S. Genome-wide target specificities of CRISPR-Cas9 nucleases revealed by multiplex Digenome-seq. Genome Res. 26, 406-415 (2016)

45. Raczy, C. et al. Isaac: ultra-fast whole-genome secondary analysis on Illumina sequencing platforms. Bioinformatics 29, 2041-2043 (2013).

46. Gresh, J., Goletz, P. W., Crouch, R. K. \& Rohrer, B. Structure-function analysis of rods and cones in juvenile, adult, and aged $\mathrm{C} 57 \mathrm{bl} / 6$ and $\mathrm{Balb} / \mathrm{c}$ mice. Vis. Neurosci. 20, 211-220 (2003).

\section{Acknowledgements}

This work was supported by the Basic Science Research Program through the National Research Foundation of Korea (NRF) funded by the Ministry of Education [2017R1A6A3A04004741 to D.H.J.], the Pioneer Research Program of NRF/MEST [2012-0009544 to J.H.K.], the Bio \& Medical Technology Development Program of the National Research Foundation and MSIP [NRF-2015M3A9E6028949 to J.H.K.], and Institute for Basic Science [IBS-R021-D1 to J.-S.K.].

\section{Author contributions}

J.-S.K. and J.H.K. supervised the research. T.K., and S.W.P., wrote the manuscript. T.K., S.W.P., D.H.J., D.K., Jin.H.K., H.-Y.C., and J.K., performed the experiments.

\section{Additional information}

Supplementary Information accompanies this paper at https://doi.org/10.1038/s41467 018-04175-y.

Competing interests: J.-S.K., T.K., D.K., and J.K. have filed a patent application based on this work under application number WO2017099494A. The remaining authors declare no competing interests.

Reprints and permission information is available online at http://npg.nature.com/ reprintsandpermissions/

Publisher's note: Springer Nature remains neutral with regard to jurisdictional claims in published maps and institutional affiliations.

Open Access This article is licensed under a Creative Commons Attribution 4.0 International License, which permits use, sharing, adaptation, distribution and reproduction in any medium or format, as long as you give appropriate credit to the original author(s) and the source, provide a link to the Creative Commons license, and indicate if changes were made. The images or other third party material in this article are included in the article's Creative Commons license, unless indicated otherwise in a credit line to the material. If material is not included in the article's Creative Commons license and your intended use is not permitted by statutory regulation or exceeds the permitted use, you will need to obtain permission directly from the copyright holder. To view a copy of this license, visit http://creativecommons.org/ licenses/by/4.0/

(C) The Author(s) 2018 